\title{
Bilateral spontaneous bisphosphonate insufficiency fractures of the proximal tibia and unilateral distal femur: a case report and recommendation
}

\begin{abstract}
Bisphosphonate insufficiency fractures of the proximal femur have been increasingly described in literature. Bilateral proximal tibial fractures due to non-traumatic loading in patients with low bone density and on long term Bisphosphonate treatment are very rare and under reported in orthopaedic literature.

We present a 77 years old Caucasian female with spontaneous onset of pain in both knees prior to presenting to casualty then to our fracture clinic. The patient is known to have Rheumatoid arthritis (RA) and has been on Bisphosphonate treatment for over 24 months following DEXA scan proven osteoporosis. Plain radiographs showed subtle linear areas of sclerosis bilaterally in her proximal tibiae. Magnetic resonance imaging (MRI) confirmed the presence of insufficiency fractures in these areas as well as her right distal femur. These fractures were treated successfully with activity modification and a hinge knee brace.

This rare presentation in a rheumatoid patient with osteoporosis and long-term bisphosphonate treatment highlights the importance of being vigilant and having a high index of suspicion for these atypical fractures around the knees and not to relate symptoms to the inflammatory process associated with rheumatoid arthritis. We think that these insufficiency fractures around the knees are under diagnosed and often confused with the inflammatory process. We would like to raise awareness and recommend MRI scan in any patient with long-term bisphosphonate treatment and a new onset of pain around the knee.
\end{abstract}

Keywords: Bisphosphonates, Insufficiency fractures, Bilateral proximal tibia, Rheumatoid Arthritis
Volume 4 Issue 2 - 2016

\author{
Zaid Al-Wattar, Ravi Kumar Kasetti \\ Trauma and Orthopaedic Department, Maidstone and Tunbridge \\ Wells NHS Trust, United Kingdom
}

\begin{abstract}
Correspondence: Zaid Al-Wattar, Orthopaedic Department, Conquest hospital, The Ridge, Saint Leonards-on-sea, East
\end{abstract} Sussex, United Kingdom, Email zaid.al-wattar@nhs.net

Received: December 20, 2015 | Published: January 21, 2016
Abbreviations: MRI, Magnetic Resonance Imaging; DEXA, Dual Energy X-ray Absorptiometry; RA, Rheumatoid Arthritis; PTH, Parathyroid Hormone

\section{Introduction}

Bisphosphonate insufficiency fractures of the proximal femur are well described in literature. This usually involves the subtrochanteric or diaphyseal region with specific documented characteristics., ${ }^{1,2}$ These fractures often result from minimal or no trauma and thought to be a consequence of lack of effective remodeling of the bone after prolonged bisphosphonate use. ${ }^{3}$ Bilateral proximal tibial fractures due to non-traumatic loading in patients with low bone density and long term Bisphosphonate treatment are very rare and under reported in orthopaedic literature.

\section{Case presentation}

We present a 77 years old Caucasian female with spontaneous onset of pain in both knees for several weeks before presenting to the casualty then to our fracture clinic. There was no history of trauma. The patient is known to have Rheumatoid arthritis (RA) for over 30 years and has been on Bisphosphonate treatment (Alendronate) for over 24 months following the diagnosis of osteoporosis by DEXA scan. Plain radiographs showed subtle linear areas of sclerosis bilaterally in her proximal tibiae (Figure $1 \&$ 2). Magnetic resonance imaging confirmed the presence of extra-articular linear high signal intensity change on T1, T2 with surrounding marrow edema consistent with insufficiency fractures in these areas as well as her right distal femur (Figure 3). These fractures were treated successfully with activity modification and weight bearing in a hinge knee brace as symptoms allows for six weeks along with physiotherapy input. Alendronate was stopped and Teriparatide (Recombinant PTH) was started.

There are very few reports of atypical insufficiency fractures involving the proximal tibia in patients on long-term Bisphosphonate therapy and this appears to be the third documented bilateral case involving the metaphyseal regions of the proximal tibia and distal right femur.

\section{Discussion}

High-quality randomized controlled trials has shown that Bisphosphonates treatment has a significant effect in reducing the risk of fractures secondary to osteoporosis. ${ }^{4}$ The treatment with Bisphosphonates has been extended to involve Paget's disease, Osteogenesis imperfecta, Steroid induced osteoporosis, secondary bone metastasis and others.

This class of drugs prevent bone mass loss by inhibiting Osteoclast bone resorption by either inhibiting Osteoclast brush boarder or inducing apoptosis depending on the presence or absence of the nitrogen atom on the Alkyl chain of the drug. ${ }^{5}$ The inhibition of Osteoclast function would interfere with the normal bone healing; suppress bone turnover and remodeling leading to a compromised biomechanical property of the bone.

When an abnormal repetitive stress or torsional force is applied to a bone with a normal biomechanical properties this would result in fatigue fractures. On the other hand, when normal stress is applied on an abnormal bone, this will result in an insufficiency fracture. ${ }^{6}$ Both types of fractures are stress fractures. In RA, insufficiency fractures are more commonly seen as a result of the osteoporosis secondary to 
the juxtra-articular bone resorption, relative immobility or the effect of corticosteroids on bone structure. In our patient, the combination of long term Bisphosphonate, Osteoporosis and RA have been recognized as possible causes for the insufficiency fractures.
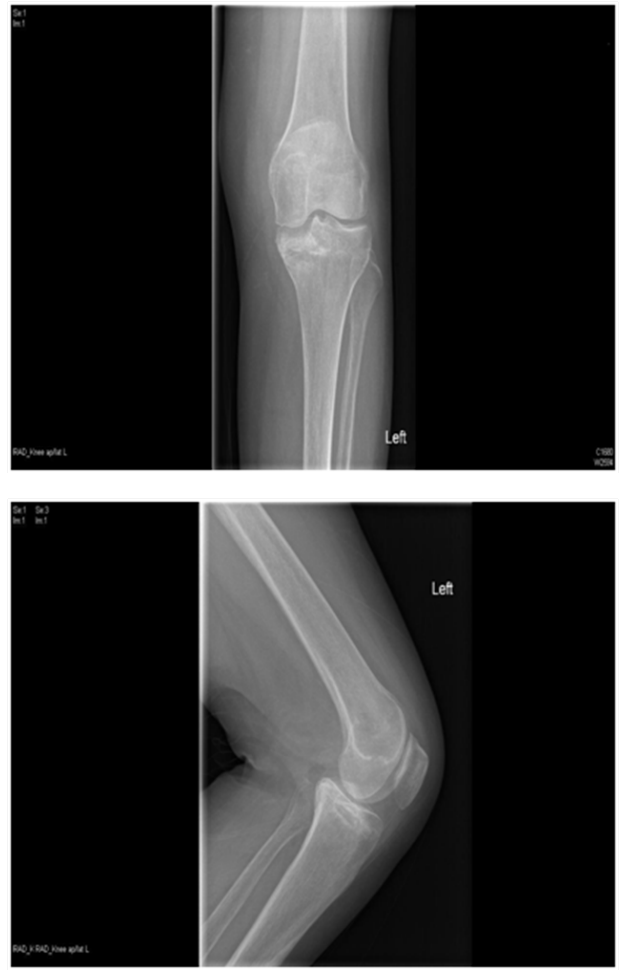

Figure I AP \& lateral radiograph of the left tibia showing an insufficiency fracture with osteosclerotic changes at the medial proximal tibia.
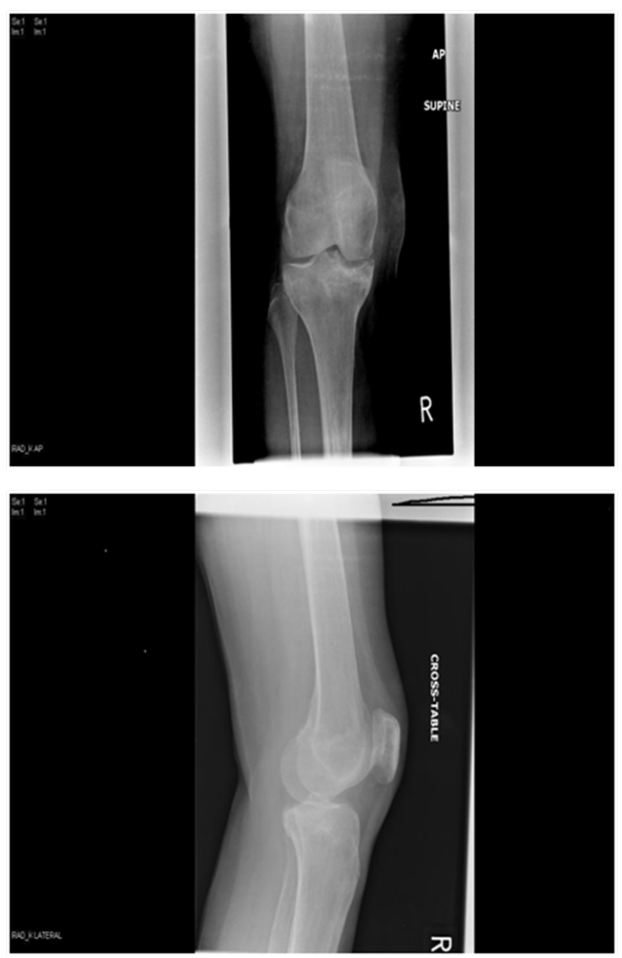

Figure 2 AP \& lateral radiograph of the right tibia and distal femur showing an insufficiency fracture with osteosclerotic changes at the medial proximal tibia and distal femur.
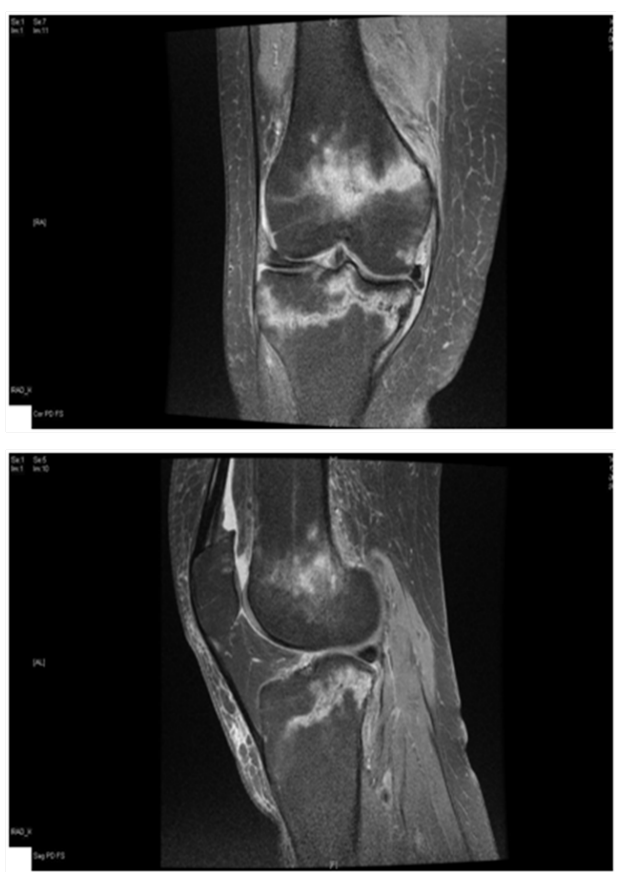

Figure 3 Magnetic resonance imaging coronal and sagittal T2 showing a linear signal change in both the proximal tibia and right distal femur with surrounding bone odema.

Few reports of atypical insufficiency fractures involving the proximal tibia in patients on long-term Bisphosphonate therapy has been published. Imbuldeniya et al. ${ }^{7}$ published a similar case which was on long term cyclical Pamidronate and successfully treated with bilateral knee supports and protected weight bearing with crutches until her symptoms resolved within two months. Vanhoof et al. ${ }^{8}$ published another case with known RA and long term cyclical Etidronate. This case was also treated conservatively.

\section{Conclusion}

This rare presentation in a rheumatoid patient with osteoporosis and long-term bisphosphonate treatment highlights the importance of being vigilant and having a high index of suspicion for these atypical fractures around the knees and not to relate symptoms to the inflammatory process associated with RA. We think that these insufficiency fractures around the knees are under diagnosed and often confused with the inflammatory process associated with RA. We would like to raise awareness and recommend MRI scan in any patient with long-term bisphosphonate treatment and a new onset of pain around the knee. We also recommend initial conservative treatment in such cases.

\section{Acknowledgments}

None.

\section{Conflicts of interest}

None.

\section{References}

1. Goh SK, Yang KY, Koh JS, et al. Subtrochanteric insufficiency fractures in patients on alendronate therapy: a caution. J Bone Joint Surg Br. 2007;89(3):349-353. 
2. Odvina CV, Zerwekh JE, Rao DS, et al. Severely suppressed bone turnover; a potential complication of alendronate therapy. $J$ Clin Endocrinol Metab. 2005;90(3):1294-1301

3. Rizzoli R, Akesson K, Bouxsein M, et al. Subtrochanteric fractures after long-term treatment with bisphosphonates: a European Society on Clinical and Economic Aspects of Osteoporosis and Osteoarthritis, and International Osteoporosis Foundation Working Group Report. Osteoporos Int. 2010;22(2):373-390.

4. Tonino RP, Meunier PJ, Emkey R, et al. Skeletal benefits of alendronate: 7 year treatment of postmenopausal osteoporotic women. Phase III Osteoporosis Treatment Study Group. J Clin Endocrinol Metab. 2000;85(9):3109-3115.
5. Morris CD, Einhorn TA. Bisphosphonates in orthopaedic surgery. $J$ Bone Joint Surg Am. 2005;87(7):1609-1618.

6. Resnick DR, Niwayama G. Diagnosis of bone and joint disorders. Saunders, Philadelphia, USA. 1995;pp.2580-2583.

7. Imbuldeniya A, Jiwa N, Murphy J. Bilateral atypical insufficiency fractures of the proximal tibia and a unilateral distal femoral fracture associated with long-term intravenous bisphosphonate therapy: a case report. Journal of Medical Case Reports. 2012;6:50.

8. Vanhoof J, Landewe $\mathrm{S}$, Vandevenne J, et al. An exceptional radiographic presentation of bilateral insufficiency fractures of the proximal tibia in a patient with rheumatoid arthritis. Ann Rheum Dis. 2003;62(3):277-279. 